\title{
Incidence of Venous Thromboembolism in Patients Living with HIV: A Cohort Study
}

\author{
Madeleine Durand, MD, MSc, ${ }^{1,2}$ Liliya Sinyavskaya, ${ }^{2}$ Yu Lan Jin, ${ }^{2}$ \\ Cécile L. Tremblay, MD, ${ }^{2,3}$ Thierry Ducruet, ${ }^{4}$ and Mikhael Laskine, MD, MSc ${ }^{1,2}$
}

To the Editor:

$\mathbf{S}^{\mathrm{E}}$ EVERAL STUDIES RAISED suspicions about risk of venous thromboembolism (VTE) in patients with HIV. Incidence of VTE in the general population has been estimated at 1-1.8 per 1000 person-years. ${ }^{1}$ In a systematic review, Klein et al. report higher incidence of VTE in HIV, ranging from $<1 \%$ to $18 \%$ in one small study of $60 \mathrm{HIV}$-positive patients. ${ }^{2}$ Several risk factors have been described for VTE in HIV, such as antiretroviral medication, ${ }^{3,4}$ chronic low-level inflammation, ${ }^{5}$ opportunistic infections, AIDS status, and cancer. ${ }^{6,7}$

To our knowledge, there is no population-based study estimating both outpatient and inpatient incidence of VTE in HIV-positive versus a comparable population of HIV-negative patients. We hypothesized that HIV-infected patients would have a higher incidence rate of both inpatient and outpatient VTE, after excluding events linked to intravenous drug use.

We conducted a retrospective cohort study using the administrative health care database of the province of Québec (RAMQ database), Canada. The database contains data on medical billing claims, hospitalization discharge summaries, and prescription drug dispensations for subscribers to the public pharmaceutical insurance (40\% of Québec population).

HIV infection was identified using ICD-9 (International Classification of Diseases, Ninth Revision) codes in physician billing claims and pharmaceutical dispensation of at least one HIV-specific antiretroviral drug. Date of first dispensation for HIV-specific drug was defined as cohort entry date. Each HIV-positive patient was matched to up to four HIV-negative patients by age, gender, and cohort entry date. Exclusion criteria were as follows: age $<20$ years at cohort entry date, no public insurance drug coverage for a year before cohort entry, and a diagnosis of atrial fibrillation or intravenous drug use in the year before cohort entry. Patients were followed into the cohort until occurrence of (1) death, (2) end of pharmaceutical insurance coverage, or (3) end of study. The study period was from January 1, 1996 to December 31, 2011.

The primary outcome was occurrence of either inpatient or outpatient VTE. Outpatient thromboembolism was defined as a new treatment with any anticoagulation drug within 15 days after a radiological test used to diagnose VTE. Inpatient thromboembolism was defined as a new diagnostic code in a hospital discharge summary. Diagnostic codes for superficial vein thrombosis and varicose veins were excluded. Thromboses were considered to be provoked if they happened (1) 30 days following discharge after major surgery; (2) current treatment with hormone replacement therapy; (3) pregnancy and postpartum period or (4) presence of active cancer. Events that did not fulfill those conditions were classified as unprovoked.

Crude and adjusted hazard ratio (aHR) of VTE were modeled using Cox proportional hazard models. All potential confounders listed in Table 1 were included in the multivariable model as time-dependent variables. All analyses were performed using SAS software version 9.3.

This research project was approved by the ethics review board of our institution.

We identified 4424 adults with HIV matched to 16,350 controls. Prevalence of each exclusion criteria was higher in the HIV-positive group; $0.5 \%$ versus $0.3 \%$ had prior diagnosis for atrial fibrillation, $1.0 \%$ versus $0.6 \%$ were anticoagulated at baseline, $2.1 \%$ versus $0.3 \%$ had prior VTE, and $11.6 \%$ versus $0.8 \%$ used intravenous drugs. The final cohort included $3820 \mathrm{HIV}$-positive and 16,074 HIV-negative participants.

Table 1 shows baseline characteristics of the study participants. Overall, mean age was 42 years and $76 \%$ were men. Cardiovascular diseases, chronic obstructive pulmonary disease, and active cancer were more prevalent in HIVpositive patients. A total of 224 incident events of VTE occurred, 87 in HIV-positive (3.59/1000 person-years, 95\% confidence interval [CI] 2.84-4.35), and 137 in HIV-negative patients (1.79/1000 person-years, 95\% CI 1.49-2.09). Overall, $121(54.0 \%)$ of events were diagnosed and treated on an outpatient basis. This proportion varied from $41.4 \%$ outpatient events in the HIV-positive to $62.0 \%$ in the HIVnegative.

Table 2 presents the results of the univariable and multivariable analyses. The aHR for VTE associated to HIV infection was 1.48 (95\% CI 1.11-1.98). Other factors independently associated with VTE were age (aHR 1.26,

\footnotetext{
${ }^{1}$ Service de Médecine interne du Centre Hospitalier de l’Université de Montréal, Montréal, Canada.

${ }^{2}$ Centre de recherche du CHUM, Montréal, Canada.

${ }^{3}$ Service de microbiologie et infectiologie du Centre Hospitalier de l'Université de Montréal, Montréal, Canada.

${ }^{4}$ Unité de recherche clinique appliquée du centre de recherche de l'hôpital Ste-Justine, Montréal, Canada.
} 
Table 1. Baseline Characteristics of Study Participants

\begin{tabular}{lcr}
\hline Characteristic & HIV-positive $(\mathrm{n}=3820)$ & HIV-negative $(\mathrm{n}=16,074)$ \\
\hline Age, years & & $41.90(0.08)$ \\
Mean (SD) & $41.76(0.17)$ & $41.00(20.00-87.00)$ \\
Median (min-max) & $41.00(20.00-86.00)$ & $12,156(75.6)$ \\
Male, $n$ (\%) & $2979(78.0)$ & \\
Follow-up duration, years & & $4.76(0.03)$ \\
Mean (SD) & $6.34(0.08)$ & $3.58(1.34-7.31)$ \\
Median (IQR) & $4.69(2.32-10.41)$ & \\
Comorbidities, $n(\%)$ & & $36(0.22)$ \\
Hepatitis-C infection & $73(1.91)$ & $154(0.96)$ \\
Alcohol abuse & $96(2.51)$ & $239(1.49)$ \\
Cardiovascular disease & $96(2.51)$ & $448(2.79)$ \\
Chronic obstructive pulmonary disease & $217(5.68)$ & $44(0.27)$ \\
Chronic kidney disease & $47(1.23)$ & $777(4.83)$ \\
Hypertension & $152(3.98)$ & $463(2.88)$ \\
Type 2 diabetes & $96(2.51)$ & $199(1.24)$ \\
Active cancer & $240(6.28)$ & \\
Medications, $n$ (\%) & & $765(4.76)$ \\
Female hormonotherapy & $104(2.72)$ & $27(0.17)$ \\
Testosterone replacement therapy & $63(1.65)$ & $48(0.3)$ \\
Antiplatelets & $13(0.34)$ & $0.12(0.00)$ \\
Number of hospitalizations, mean (SD) & $0.55(0.01)$ & \\
\hline
\end{tabular}

Characteristics are measured in the year before cohort entry date. $\mathrm{IQR}$, interquartile range; SD, standard deviation.

95\% CI 1.11-1.43 for each 10-year increase in age), cardiovascular disease (aHR 1.53, 95\% CI 1.02-2.29), chronic kidney disease (aHR 2.41, 95\% CI 1.42-4.11), active cancer (aHR 4.99, 95\% CI 3.35-7.43), current exposure to female hormonotherapy (aHR 2.99, 95\% CI 1.57-5.69), and being within 30 days of a surgery (aHR 49.99, 95\% CI 34.09-73.3).

We found that HIV infection was associated to a $1.48(95 \%$ CI 1.11-1.98) increase in the incidence of inpatient and outpatient VTE, compared with an age- and gender-matched HIV-negative population. Other factors independently associated with an increased risk of VTE in our cohort were age, being diagnosed with cardiovascular disease, chronic kidney disease, active cancer, being exposed to female hormonotherapy, and postsurgical period.

These results are consistent with prior data. Rasmussen et al. examined the risk of inpatient VTE in people living with HIV compared with matched HIV-negative patients. They found an incidence rate ratio of 3.84; 95\% CI 2.99-4.93 associated with HIV infection. ${ }^{8}$ Fultz et al. reported standardized incidence ratios for VTE in HIV patients of $1.39 ; 95 \%$ CI 1.26-1.52 before 1996 (inpatient events only) and $1.33 ; 95 \%$ CI 1.24-1.43 after 1996 (both inpatient and outpatient). ${ }^{9}$ Malek et al. reported odds ratios for VTE in HIV-positive patients of $1.43 ; 95 \%$ CI $1.39-1.46 .^{10}$ Other studies that also reported increased risk of VTE in HIV were mainly case reports, case series, and reviews of hospital discharge databases ${ }^{4,10-12}$

Key limitations of our study include data limitations. We had no access to laboratory data (viral load and CD4 count). Although this would have allowed us to characterize further the pathways that lead to increased thrombotic risk in patients living with HIV, it does not interfere with the interpretation of our results. Data on some potentially important confounders are lacking, such as smoking and obesity. Unmeasured confounders could explain some of the association found. Our study was conducted using the administrative health care databases of the province of Québec, Canada. This is a developed country setting with universal health care coverage. The external validity of our results might be low in other socioeconomic settings.

Strengths of this study include its large sample size, its control group, and the inclusion of both inpatients' and outpatients' events. Because outpatients' codes are generated as part of physician's billing, it is possible that a physician would write down the code for thrombophlebitis for a suspicion of thrombophlebitis, creating falsely high event rates (false positives). To avoid this, we used a stringent outpatient event definition, requiring an imaging test used for VTE diagnosis, followed by initiation of anticoagulation. This will limit the possibility of false positive events. Inpatient diagnostic codes are generally more valid, as they are written by the treating physician at the time of hospital discharge, after complete investigation. We are, therefore, confident that our outcome definition is valid, and that any outcome misclassification would not be differential in the HIV-positive and HIV-negative.

In conclusion, we found that HIV infection was associated with increased risk of VTE, even after adjustment for confounders. Medical practitioners should be aware of that association when diagnosing and treating thromboembolic disease.

\section{Declaration}

M.D. and T.D. had full access to all the data in the study and take responsibility for the integrity of the data and the accuracy of the data analysis.

\section{Authors' Contributions}

M.D. wrote the scientific protocol, planned and oversaw the analysis. M.D. and L.S. wrote the article's first draft. T.D. and Y.J. reviewed the scientific protocol, participated in 
Table 2. Univariable and Multivariable Hazard Ratios for First Venous Thromboembolism (Outpatient and InPatient) According to HIV Status and Covariates

\begin{tabular}{|c|c|c|c|}
\hline Characteristic & $\begin{array}{l}\text { Number of events } \\
\quad(\mathrm{n}=224)\end{array}$ & $\begin{array}{l}\text { Crude hazard ratio } \\
\quad(95 \% \text { CI })\end{array}$ & $\begin{array}{c}\text { Adjusted hazard ratio } \\
(95 \% \text { CI })\end{array}$ \\
\hline \multicolumn{4}{|l|}{ HIV } \\
\hline Positive & 87 & $2.01(1.57-2.55)$ & $1.48(1.11-1.98)$ \\
\hline Negative & 137 & Referent & Referent \\
\hline \multicolumn{4}{|l|}{ Sex } \\
\hline Male & 175 & $1.03(0.78-1.36)$ & $1.17(0.82-1.67)$ \\
\hline Female & 49 & Referent & Referent \\
\hline Age (per 10 years increase in age) & N/A & $1.39(1.25-1.54)$ & $1.26(1.11-1.43)$ \\
\hline \multicolumn{4}{|l|}{ Hepatitis C infection ${ }^{\mathrm{a}}$} \\
\hline Positive & 12 & $2.51(1.53-4.14)$ & $1.58(0.85-2.92)$ \\
\hline Negative & 212 & Referent & Referent \\
\hline \multicolumn{4}{|l|}{ Alcohol abuse $^{\mathrm{a}}$} \\
\hline Present & 23 & $2.26(1.55-3.3)$ & $1.2(0.76-1.91)$ \\
\hline Absent & 201 & Referent & Referent \\
\hline \multicolumn{4}{|l|}{ Cardiovascular disease $^{\mathrm{a}}$} \\
\hline Present & 38 & $2.65(1.92-3.66)$ & $1.53(1.02-2.29)$ \\
\hline Absent & 186 & Referent & Referent \\
\hline \multicolumn{4}{|l|}{ Chronic obstructive lung disease ${ }^{\mathrm{a}}$} \\
\hline Present & 39 & $1.93(1.41-2.64)$ & $1.34(0.92-1.94)$ \\
\hline Absent & 185 & Referent & Referent \\
\hline \multicolumn{4}{|l|}{ Chronic kidney disease $^{\mathrm{a}}$} \\
\hline Present & 18 & $4.27(2.71-6.72)$ & $2.41(1.42-4.11)$ \\
\hline Absent & 206 & Referent & Referent \\
\hline \multicolumn{4}{|l|}{ Hypertension $^{\mathrm{a}}$} \\
\hline Present & 41 & $1.43(1.04-1.96)$ & $0.95(0.65-1.39)$ \\
\hline Absent & 183 & Referent & Referent \\
\hline \multicolumn{4}{|l|}{ Type 2 diabetes $^{\mathrm{a}}$} \\
\hline Present & 19 & $1.37(0.92-2.04)$ & $0.65(0.39-1.08)$ \\
\hline Absent & 205 & Referent & Referent \\
\hline \multicolumn{4}{|l|}{ Active cancer ${ }^{\mathrm{b}}$} \\
\hline Present & 63 & $16.15(12.09-21.58)$ & $4.99(3.35-7.43)$ \\
\hline Absent & 161 & Referent & Referent \\
\hline \multicolumn{4}{|l|}{ Antiplatelet medication ${ }^{\mathrm{b}}$} \\
\hline Present & 9 & $3.02(1.53-5.95)$ & $0.84(0.33-2.12)$ \\
\hline Absent & 215 & Referent & Referent \\
\hline \multicolumn{4}{|l|}{ Female hormonotherapy ${ }^{c}$} \\
\hline Present & 17 & $2.54(1.61-4.0)$ & $2.99(1.57-5.69)$ \\
\hline Absent & 207 & Referent & Referent \\
\hline \multicolumn{4}{|l|}{ Testosterone replacement therapy $\mathrm{y}^{\mathrm{c}}$} \\
\hline Present & 11 & $1.89(1.0-3.58)$ & $0.66(0.24-1.81)$ \\
\hline Absent & 213 & Referent & Referent \\
\hline \multicolumn{4}{|l|}{ Postsurgical period ${ }^{\mathrm{d}}$} \\
\hline Present & 67 & $93.48(69.03-126.59)$ & $49.99(34.09-73.3)$ \\
\hline Absent & 157 & Referent & Referent \\
\hline
\end{tabular}

${ }^{a}$ Defined as time-dependent variables during the entire follow-up time, once present, presence of the characteristic is considered irreversible for remainder of follow-up time.

${ }^{\mathrm{b}}$ One-year period after any diagnostic code for cancer.

${ }^{c}$ Defined as current use of medication: dispensation date+duration of dispensation +30 days.

${ }^{\mathrm{d}}$ Defined as 30 days after hospitalization discharge for a surgical procedure either intra-abdominal or orthopedic of lower limb or spine, proximal to and including ankle.

CI, confidence interval.

analysis planning, conducted the data analysis, and reviewed the article. M.L. and C.T. reviewed the protocol and article for important intellectual content.

\section{Author Disclosure Statement}

M.D., T.D., L.S. and M.L. have no conflict of interest to declare. C.L.T. has no conflict of interest to declare that relate to this study. She receives honorarium or research funding from Merck, VIIV, and Gilead in form of research grants, speaker, and advisory board member.

\section{Funding Information}

M.D. is supported by a clinician-researcher salary award from the Fonds de recherche du Québec-Santé, which 
partially funded this study. Funds to acquire data for this study were acquired in part by an unrestricted educational grant from Boerhinger-Ingelheim granted to Cécile L. Tremblay in 2007, and in part by an operating grant from the Canadian Association for AIDS research (Grant No. 023 501) granted to M.D. in 2010.

\section{References}

1. Heit JA. Epidemiology of venous thromboembolism. Nat Rev Cardiol 2015;12:464-474.

2. Klein SK, Slim EJ, de Kruif MD, et al. Is chronic HIV infection associated with venous thrombotic disease? A systematic review. Neth J Med 2005;63:129-136.

3. Majluf-Cruz A, Silva-Estrada M, Sanchez-Barboza R, et al. Venous thrombosis among patients with AIDS. Clin Appl Thromb Hemost 2004;10:19-25.

4. Matta F, Yaekoub AY, Stein PD. Human immunodeficiency virus infection and risk of venous thromboembolism. Am J Med Sci 2008;336:402-406.

5. O'Brien MP, Zafar MU, Rodriguez JC, et al. Targeting thrombogenicity and inflammation in chronic HIV infection. Sci Adv 2019;5:eaav5463.

6. Kiser KL, Badowski ME. Risk factors for venous thromboembolism in patients with human immunodeficiency virus infection. Pharmacotherapy 2010;30:1292-1302.

7. Epaulard O, Foote A, Bosson JL. Chronic infection and venous thromboembolic disease. Semin Thromb Hemost 2015;41:644-649.
8. Rasmussen LD, Dybdal M, Gerstoft J, et al. HIV and risk of venous thromboembolism: A Danish nationwide population-based cohort study. HIV Med 2011;12:202210.

9. Fultz SL, McGinnis KA, Skanderson M, Ragni MV, Justice AC. Association of venous thromboembolism with human immunodeficiency virus and mortality in veterans. Am J Med 2004;116:420-423.

10. Malek J, Rogers R, Kufera J, Hirshon JM. Venous thromboembolic disease in the HIV-infected patient. Am J Emerg Med 2011;29:278-282.

11. Howling SJ, Shaw PJ, Miller RF. Acute pulmonary embolism in patients with HIV disease. Sex Transm Infect 1999;75:25-29.

12. Copur AS, Smith PR, Gomez V, Bergman M, Homel $\mathrm{P}$. HIV infection is a risk factor for venous thromboembolism. AIDS Patient Care STDS 2002;16:205-209.

Address correspondence to: Madeleine Durand, $M D, M S c$ Centre de recherche $\mathrm{du}$ CHUM

Room SO1-134

850, rue Saint-Denis Montréal, QC H2X OA9

Canada

E-mail: madeleine.durand.chum@ssss.gouv.qc.ca 